
allemande

47-2 | 2015

Retour sur le modèle Rhénan : humanisme, capitalisme et métropolisation | Patrimonialisation du passé « allemand » en Europe centrale après 1990

\title{
Cultures régionales et développement économique dans l'espace rhénan et au-delà
}

Autour de la parution de deux ouvrages

René Kahn

\section{(2) OpenEdition}

Édition électronique

URL : https://journals.openedition.org/allemagne/290

DOI : $10.4000 /$ allemagne.290

ISSN : 2605-7913

Éditeur

Société d'études allemandes

\section{Édition imprimée}

Date de publication : 16 décembre 2015

Pagination : 293-305

ISSN : 0035-0974

\section{Référence électronique}

René Kahn, «Cultures régionales et développement économique dans l'espace rhénan et au-delà », Revue d'Allemagne et des pays de langue allemande [En ligne], 47-2 | 2015, mis en ligne le 13 décembre 2017, consulté le 19 mai 2021. URL : http://journals.openedition.org/allemagne/290 ; DOI : https:// doi.org/10.4000/allemagne.290 


\section{Cultures régionales et développement économique dans l'espace rhénan et au-delà}

\section{Autour de la parution de deux ouvrages}

- René Kahn *

\section{Introduction}

L'une des vocations de l'APR ${ }^{(1)}$ est certainement de s'intéresser aux spécificités du modèle économique et social du Rhin supérieur et plus largement de l'espace rhénan. De mon côté, je m'intéresse aux rapports entre culture et économie dans les modèles régionaux de développement. Il s'agit selon les cas de rapports d'opposition, de complémentarité ou de coopération. Je vais montrer que les deux préoccupations se rejoignent. Un ouvrage collectif publié sous la direction de Pierre Lamard et Nicolas Stoskopf, en hommage aux travaux de l'historien Michel Hau et consacré à l'entreprise rhénane ${ }^{(2)}$, vient relancer opportunément la question des singularités du tissu économique régional rhénan et de l'existence de caractéristiques culturelles dans la façon d'organiser la vie économique.

\section{Retour sur l'entreprise rhénane}

Il s'agit d'un ouvrage en trois parties (Comparaisons d'écosystèmes / L'observatoire alsacien / Destins d'entreprises), comportant des monographies. Comme

* Maître de conférences HDR en économie à l'Université de Strasbourg. Chercheur au Bureau d'Économie théorique et appliquée (BETA, UMR 7522); membre de l'Observatoire des Politiques économiques en Europe (OPEE).

1 Association de Prospective Rhénane. <http://www.apr-strasbourg.org/2-presentation-de-l-apr.html>. Le présent article est tiré d'une conférence donnée par l'auteur à l'APR le 24 juin 2015. Je remercie son président, le professeur Jean-Alain Héraud, ainsi que le professeur Florence Rudolf pour m'avoir aidé, l'un et l'autre, à finaliser cet article.

2 Pierre Lamard et Nicolas Stoskopf (dir.), L'entreprise rhénane, mythe ou réalité? Ouvrage publié en hommage à Michel Hau: actes des cinquièmes Journées d'histoire industrielle de Mulhouse et de Belfort, octobre 2012, Paris, Picard, 2015. 
pour tout ouvrage de ce type, les contributions sont diverses (du fait du statut des auteurs, des méthodes d'approches) mais il y a une interrogation commune autour de l'entreprise rhénane, qu'on peut formuler ainsi: existe-t-il dans certaines régions un modèle économique spécifique de type rhénan? Ou encore notre région est-elle encore influencée par un modèle d'organisation germanique des entreprises et des activités économiques, ce modèle pouvant tour à tour s'appeler capitalisme rhénan, Mittelstand, modèle macroéconomique allemand, ordolibéralisme, économie sociale de marché, entreprise rhénane, capitalisme familial, économie de marché coordonnée, etc. ? Évidemment chacun de ces modèles renvoie à une histoire et des références théoriques précises. Mais pour tirer cela au clair je vais commencer par évoquer les travaux de l'économiste hongrois Karl Polanyi.

\section{Une hypothèse de Karl Polanyi utile pour comprendre l'actualité du modèle rhénan}

Au-delà de l'étude des spécificités de l'entreprise rhénane dont nous allons parler, cet ouvrage prolonge implicitement les travaux de la période américaine de l'économiste hongrois Karl Polanyi (1947-1964) sur les rapports de l'économie de marché au reste de la société, sur la place des institutions économiques marchandes par rapport à l'économie réelle et par rapport aux autres dimensions de l'organisation sociale. Plus précisément Polanyi pose la question de savoir si dans une économie capitaliste et plus encore dans une société de marché, les raisonnements tenus sont suffisants pour satisfaire les exigences de viabilité de la société et de subsistance matérielle des individus.

Rappelons pour faire court que Polanyi a décrit le processus par lequel la sphère économique marchande s'autonomise (désencastrement) et envahit le domaine initialement plus large des rapports des hommes avec la nature et des hommes entre eux. De ce fait la sphère économique marchande néglige les autres rationalités construites ou héritées (culturelles) en installant l'utilitarisme, la concurrence et la motivation du profit comme les seules références nécessaires pour régler tous les problèmes économiques. Polanyi pensait que ce désencastrement et l'assimilation de l'économie de marché à toute l'économie constituaient une erreur grave, une illusion inefficace qui conduit au paradoxe suivant: plus on se focalise sur la dimension marchande de l'économie moins on est en mesure de résoudre les problèmes économiques. Il le dit explicitement dans la préface de La subsistance de l'homme ${ }^{(3)}$ : Polanyi n’est pas le seul

3 « Notre pensée sociale, dans la mesure où elle est entièrement concentrée sur la sphère économique, est mal équipée pour affronter les exigences économiques de notre époque en peine adaptation. Il est difficile, sinon impossible, pour une société centrée sur le marché comme la nôtre d'évaluer les limites de l'importance de l'économique. En effet une fois que les activités quotidiennes de l'homme ont été organisées par divers types de marchés, fondées par les motivations du profit, déterminées par des comportements concurrentiels, et orientées selon une échelle de valeurs utilitariste, la société devient un organisme qui, dans toutes ses dimensions essentielles, est soumis à des objectifs lucratifs. Ayant ainsi absolutisé en pratique la motivation du gain économique, l'homme perd la capacité de relativiser mentalement. Son imagination est bridée par des limites étouffantes [...] il a l'habitude de qualifier d'économiques un ensemble de motivations particulières, d'attitudes caractéristiques et d'objectifs très spécifiques qui ne sont que des accessoires de l'économie réelle, ne devant leur existence qu'à l'interaction éphémère de traits culturels [...]. Cette mentalité dépassée du marché est, à mon avis, l'obstacle principal à une approche réaliste des problèmes économiques de l'époque qui s'ouvre devant nous. » Karl Polanyi, La subsistance de l’homme, Paris, Flammarion, 2011, p. 19. 
économiste à s'être intéressé à l'anthropologie économique, aux représentations de la vie économique et aux modèles les plus adéquats (Karl Marx, Max Weber, Thornstein Veblen, John Maynard Keynes, François Perroux, Amartya Sen, Jacques Généreux, Emmanuel Todd l'ont fait également) mais il est peut-être le seul à avoir qualifié de culturel l'ensemble des institutions de l'économie de marché.

Cela va peut-être vous surprendre mais il me semble que les réflexions sur le modèle rhénan et l'entreprise rhénane relèvent du même questionnement. Les directeurs de l'ouvrage, Pierre Lamard et Nicolas Stoskopf, n'écrivent-ils pas en introduction, à propos de Michel Hau, de sa thèse sur L'industrialisation de l'Alsace (1803-1839): «Il constate qu'une grande partie de la croissance économique de l'Alsace s'explique par des phénomènes qui n'appartiennent pas principalement au domaine des sciences économiques, comme la progression de l'alphabétisation, la permanence des structures familiales et l'évolution des croyances religieuses. Cette idée est fondamentale dans la genèse d'un programme de recherche qu'il mettra ensuite en œuvre tout au long de sa carrière " ${ }^{(4)}$. Les auteurs vont rappeler quels sont ces facteurs qui sont la face cachée de la performance économique des régions de l'Europe rhénane (y compris actuellement dans la mondialisation, d'où l'actualité de cette réflexion), facteurs qui sont pour certains clairement économiques alors que d'autres relèvent davantage de la "mentalité ", des caractères communs, des comportements spécifiques à ces régions, en un mot de la culture régionale rhénane. Citons quelques-uns de ces caractères: la stabilité des institutions et leur inscription dans la longue durée (à travers le rôle de la famille, les dynasties d'entrepreneurs, la transmission), l'importance accordée à la ressource humaine comme facteur de compétitivité (éducation, formation professionnelle, ouverture à l'innovation), la capacité d'adhésion au groupe, la valorisation de l'activité productive (par rapport à l'activité spéculative), l'autofinancement et le recours aux banques plutôt qu'au marché financier, le faible coût du travail, la nécessité d'exporter, la décentralisation et le faible rôle de l'État, l'ancrage territorial avec un mélange de coopération interentreprises et de concurrence au sein de districts industriels avant la «mode» partie des travaux de Becattini sur la $3^{\mathrm{e}}$ Italie $^{(5)}$.

Cet ouvrage sur l'entreprise rhénane fait évidemment écho à celui de Michel Albert, Capitalisme contre capitalisme (1991) qui oppose le capitalisme rhénan au capitalisme anglo-saxon (court termiste, anonyme, etc.) à cette différence essentielle près que l'ouvrage d'aujourd'hui s'attache moins à décrire un modèle macro-économique germanique et ses réformes structurelles (l'économie sociale de marché à l'allemande et ses évolutions depuis 1948) qu'un écosystème d'entreprises qui n'est pas seulement constitué d'entreprises familiales de taille moyenne (du Mittelstand) parce qu'on retrouve aussi ces caractéristiques dans certaines très grandes entreprises. L'ouvrage sur l'entreprise rhénane démontre non seulement la supériorité économique de cet écosystème d'entreprises, en termes de compétitivité, d'innovation, d'emploi, etc. mais aussi sa capacité à affronter la mondialisation, à surmonter la crise, et même

4 Lamard/Stoskopf (dir.), L'entreprise rhénane (note 2), p. 12.

5 Giacomo Becattini est l'un des pères de l'école italienne d'économie industrielle. Il a réactualisé l'intuition marshallienne de «l'atmosphère industrielle » favorable aux firmes, associée à une concentration géographique et une organisation des systèmes socio-productifs territorialisés de l'Italie du Centre et du Nord-Est. 
à faire société (de par sa capacité à créer du lien, familial, territorial, social) tout en s'interrogeant sur sa capacité d'adaptation et d'évolution au contexte actuel. Le débat que ce livre ouvre et auquel ont participé vingt-cinq auteurs sans compter les auteurs cités dans les bibliographies (d'où l'impossibilité de résumer les arguments) est très riche mais j'en retiendrai trois aspects principaux sous forme de questions:

- Ce modèle est-il bien réel? (On peut discuter de la manière de comprendre cette question.) Quelles en sont les caractéristiques? Est-il d'actualité, est-il vivant (et pas seulement historique et fossilisé car il y a beaucoup de travaux historiques sur l'entreprise rhénane au XIX ${ }^{\mathrm{e}}$ siècle). Plus largement, ce modèle a-t-il encore un avenir?

- Il a pu essaimer en Alsace, est-il éventuellement transposable en France?

- De quoi est-il constitué: de valeurs culturelles essentiellement territoriales ou davantage, d'un pragmatisme économique?

Ces trois familles de questions nous ramènent au soubassement territorial et culturel de l'organisation économique, et plus particulièrement la troisième pose explicitement la question de la place de la culture régionale dans l'organisation économique. Je vais développer un peu les réponses apportées.

\section{Trois séries de questions autour de l'entreprise rhénane}

a. Réalité et actualité du modèle rhénan?

Il y a effectivement des entreprises qui fonctionnent suivant des règles distinctes de celles du capitalisme anglo-saxon et ce modèle retrouve une actualité comme en témoignent de nombreuses publications et articles dans la presse ${ }^{(6)}$.

Il y a actuellement un regain d'intérêt pour le modèle rhénan. Les difficultés actuelles, la prise de conscience des limites du système pour les individus, les sociétés et pour la planète (épuisement des ressources, changement climatique) font que beaucoup de monde est à la recherche d'un nouveau modèle de développement. Le modèle rhénan séduit parce qu'il est humaniste, écologique, mais peut-être aussi par son ambiguïté. Ce qui fait le succès du modèle rhénan c'est qu'il permet de réunir plusieurs conceptions de l'économie et de l'entreprise rhénane (qu'on retrouve dans le livre) et des conceptions passablement contradictoires. Par exemple une version humaniste (qui met l'accent sur les valeurs, l'importance de la cohésion sociale, la participation), une version paternaliste (l'entreprise au centre de l'organisation sociale, acteur principal du changement et source d'inspiration des politiques publiques), une version néolibérale (comportant elle-même des variantes). Une variante ordolibérale où l'État pose les règles du jeu économique mais s'interdit d'intervenir directement et de réguler, laissant les entreprises, les corps intermédiaires, les institutions locales et régionales se débrouiller et une variante plus franchement hayekienne, qui dénonce les dangers du keynésianisme, et généralise la société de marché. Le modèle allemand (souvent qualifié de rhénan) ne convainc pas nécessairement ou complètement mais il faut distinguer entre modèle macro-économique allemand, ordolibéralisme, entreprise rhénane, économie sociale de marché, etc. Ce n’est pas seulement le modèle rhénan

6 Gabriel Colletis, "Mutation du modèle rhénan et avenir du modèle européen ", Regards sur l'économie allemande, Bulletin économique du CIRAC, $n^{\circ} 67$ (2004), p. 7-14; Guillaume DuvAL, Made in Germany. Le modèle allemand au-delà des mythes, Paris, Seuil, 2013; Bruno Odent, Modèle allemand, une imposture. L'Europe en danger, Montreuil, Le temps des cerises, 2013. 
qui est intéressant mais la poussée intellectuelle pour voir émerger dans le monde un nouveau modèle de développement.

\section{b. La "transposabilité » du modèle rhénan?}

La question de la transposition possible du modèle rhénan est au cour du questionnement de l'ouvrage. Compte tenu du succès économique de l'Allemagne, chacun s'interroge sur les facteurs de ce succès. On montre qu'il y a clairement deux analyses opposées: un groupe de chercheurs considère que le contexte culturel et territorial n'a rien à voir dans les performances de l'économie allemande, il s'agit exclusivement de facteurs, de choix et de stratégie économiques, auquel cas rien ne s'oppose à la transposition du modèle, il suffirait d'imiter les Allemands. C'est le point de vue d'Alain Fabre qui dit explicitement: «Le point le plus important consiste à considérer que le modèle rhénan relève de la stratégie, non du culturel ${ }^{(7)}$. C’est aussi dans ce même ouvrage le point de vue de Patrice Charlier qui montre qu'à travers les théories de l'entreprise dites partenariale, de l'intendance et cognitive, on peut montrer que les aspects culturels comme l'altruisme dans le cadre familial ou la conception partenariale qui caractérise l'entreprise rhénane sont encore fondés sur la rationalité économique. À terme, l'entreprise est gagnante si le management et les actionnaires se comportent ainsi. Les traits culturels s'expliquent par la rationalité économique.

A contrario, pour Pierre Lamard et Nicolas Stoskopf ou encore Luc Julien-SaintAmand $^{(8)}$, et d'autres auteurs sur le même sujet, les traits économiques s'expliquent par la culture et le territoire. Le modèle n'est donc pas transposable. L'entreprise est indissociable de son milieu (de l'histoire, des croyances, des traditions, etc.). Cela peut nous amener à considérer comme Philippe Aydalot (l'un des fondateurs de la science régionale en France) que les entreprises sont dans beaucoup de dimensions une émanation directe $\mathrm{du}$ territoire. Ce sont les territoires qui entreprennent et innovent. D'où le concept de milieu innovateur ${ }^{(9)}$. Guillaume Duval, dans Made in Germany. Le modèle allemand au-delà des mythes, veut surtout démystifier le modèle (ce ne sont pas les lois Hartz et la politique de rigueur qui caractérisent le modèle allemand mais sa longue tradition de corporatisme, de coopération et de codétermination). Brunot Odent ${ }^{(10)}$ met en garde les Européens, Gabriel Colletis ${ }^{(11)}$ anticipe l'explosion du modèle allemand et Johann Chapoutot $^{(12)}$ rappelle ses vertus démocratiques et sa capacité de renouvellement.

7 Alain FABRE, «Une stratégie de croissance pour la France, les leçons du modèle rhénan », in: LAMARD/ Sтовкорғ (dir.), L'entreprise rhénane (note 2), p. 37.

8 «L'entreprise rhénane est une réalité..., une réalité rhénane », in: ibid., p. 43.

9 «L'entreprise n'est pas un agent innovateur isolé, elle est partie du milieu qui la fait agir. Le passé des territoires, leur organisation, leurs comportements collectifs, le consensus qui les structure sont des composantes majeures de l'innovation. » Philippe Ay dalot, Milieux innovateurs en Europe (1986), in: Andrée Matteaccioli (dir.), Philippe Aydalot pionnier de l'économie territoriale, Paris, L'Harmattan, 2004, p. 382.

10 Modèle allemand (note 6).

11 «Mutation du modèle rhénan» (note 6).

12 Johann Снарочтот, "Le véritable modèle allemand, c'est celui de l'écologie politique ", Le Monde, mercredi 19 novembre 2014, p. 15. 


\section{c. Quelle est la nature du modèle rhénan?}

La troisième question relative à la nature du modèle revient à s'interroger sur le rôle que peut jouer la culture régionale dans le développement économique des territoires. Ainsi l'hypothèse culturaliste bien que très contestée (y compris dans cet ouvrage) fait moins peur ou paraît plus recevable aujourd'hui. C'est l'occasion pour moi d'évoquer un travail complémentaire et la parution d'un deuxième ouvrage consacré à l'étude des relations entre les cultures régionales et le développement économique. Le livre est le résultat d'un congrès (le $4^{\mathrm{e}}$ depuis 2000) qui s'est tenu à Marseille en 2013 et qui a réuni les contributions d'une petite vingtaine de chercheurs mais aussi de chefs d'entreprise, d'associations et d'élus ${ }^{(13)}$.

L'ouvrage est divisé en quatre parties: une partie théorique; une partie consacrée à des terrains régionaux; une troisième partie sur les interfaces métiers d'art/ territoires et enfin une quatrième partie qui regroupe les témoignages des chefs d'entreprise, des associations (en particulier de l'Union Provençale et de la fondation Louis Vouland) et d'élus de la région Provence-Alpes-Côte d'Azur.

Pour comprendre de quoi il s'agit, il faut un peu de patience, il faut refreiner un besoin d'objectivité cartésienne car le terme de culture renvoie très souvent à tout ce qu'on ne peut pas expliquer et par conséquent tout ce pour quoi un chercheur doit montrer un peu de méfiance s'il veut être pris au sérieux. Le terme de culture est tellement polysémique et général (André Gorz donnait de la culture une définition anthropologique très concise: c'est l'humanité de l'homme) qu'il ne faut pas chercher à circonscrire tout ce qu'il englobe: culture populaire, arts, sciences, langages, pratiques, coutumes, savoir-faire, traditions, etc. La culture, c'est tout cela et bien d'autres choses encore. Il y a partout de la culture et la culture est dans tout. C'est la raison pour laquelle, d'une part on ne s'est pas intéressé principalement à la culture en général mais aux cultures régionales, et d'autre part nous avons travaillé sur les rapports cultures régionalesdéveloppement économique. Je veux d'abord souligner le statut particulier de cette recherche et de son objet.

\section{Au-delà du modèle rhénan, les rapports cultures régionales-développement économique}

\section{Un objet singulier à plusieurs titres}

D’un point de vue épistémologique nous avons affaire à un objet singulier. L'étude des relations développement économique-cultures régionales ne s'apparente pas aux objets usuels de recherche en sciences sociales. La raison principale est qu'il ne s'agit pas de mettre en relation deux ou plusieurs variables à partir d'un modèle et de tester la valeur du modèle à partir de mesures quantitatives comme on le fait le plus souvent en analyse économique ou en sociologie lorsqu'on veut expliquer un phénomène, mais d'approfondir la compréhension des relations entre deux notions très larges et polysémiques:

- la notion de développement économique qui interroge le modèle de développement régional (capitaliste, mais plus ou moins, endogène, exogène, innovant, durable, etc.) et ses finalités (le bien-être du plus grand nombre ou l'enrichissement de quelques-uns, le

13 René KAhn, Roseline Le SQuère et Jean-Michel Kosianski (dir.), Cultures régionales, développement économique. Des ressources territoriales pour les économies régionales, Paris, L’Harmattan, 2014. 
rayonnement international ou l'ancrage des populations et des activités). L'économie politique distingue clairement les notions de croissance (l'augmentation du PIB) et de développement. Cette dernière notion implique aussi les capacités et l'épanouissement des individus comme de la collectivité, la redistribution, l'entretien des ressources collectives, la cohésion territoriale, etc.

- les notions de culture et de culture régionale à l'interface du singulier et de l'universel.

Dans cet ouvrage, les cultures régionales ne sont pas principalement définies par des facteurs communautaires et identitaires, ni par un folklore figé mais plutôt comme un aspect du développement et de l'organisation sociale des territoires qui échappe aux critères et méthodes usuelles de l'analyse économique. Les notions de culture et de culture régionale interrogent des modes de pensée et d'organisation à la fois singuliers et universels (l'universalité est une condition pour la culture de pouvoir s'adresser au plus grand nombre même lorsqu'elle montre une origine territoriale). Mes collègues et moi-même nous intéressons à des singularités, des spécificités territoriales et à la façon dont ces spécificités interfèrent avec le développement économique (comme dans le livre précédent sur l'entreprise rhénane). La culture est ici conçue comme un pôle, un attracteur, un système de valeurs qui dans toute société se distingue de l'économie et dialogue avec elle.

L'interface économie-cultures régionales mobilise d'autres temporalités.

Habituellement, la plupart des problèmes régionaux sont traités dans l'urgence. Nous pensons au contraire que ces interfaces exigent du temps, de la concertation et de la réflexion. Il s'agissait de comprendre les problèmes des collectivités locales, les mutations en cours dans les régions étudiées. Les interfaces les plus connues sont les industries culturelles, les objets d'art, le capital social, les fêtes et les traditions qui présentent à la fois un aspect économique et un aspect culturel. Ce sont celles qui ont déjà un caractère économique. Il y en a d'autres qui concernent les formes culturelles non intégrées à l'économie.

Le plus souvent, les institutions de développement (OCDE, Commission européenne, Banque mondiale, etc.) et les chercheurs en économie, conscients du pouvoir extrinsèque de la culture (de sa capacité à fertiliser l'économie/fertilisation croisée), se posent la question dans les termes suivants: comment mettre de la culture dans les processus productifs et les échanges pour les rendre plus efficaces? Comment mettre la science, l'innovation, les institutions (régionales), la créativité, au service du développement, c'est-à-dire au service de la création de valeur et éventuellement au service de la production, de l'emploi, des exportations, etc. ? The European Statistical System Network on Culture d'EUROSTAT montre que dix secteurs culturels importants (industries culturelles): arts vivants, artisanat, audiovisuel, presse, architecture, arts visuels, etc. interviennent au niveau de six fonctions économiques fondamentales (la création, la production, la diffusion et le commerce, la conservation, l'éducation, la formation-management) qui concernent tous les secteurs d'activités. La culture (artistique, scientifique, linguistique, etc.) ne s'accommode pas nécessairement d'un rôle fixé à l'avance. Elle interagit, transforme, reformule les questions. La culture est partout dans toutes les réalisations et toutes les activités humaines. Elle affecte en général positivement (sauf dérives aberrantes dans les sociétés totalitaires: l'art nazi ou la biologie de Lyssenko) et dynamise tout ce qu'elle touche. C'est ce que nous montrons nous aussi dans ce livre. On peut s'en féliciter. A priori cela ne pose pas de problème. 
Quelques enseignements tirés de cet ouvrage collectif:

Comme pour l'ouvrage précédent la diversité des points de vue est grande mais ce qui en fait l'unité c'est l'importance que chacun des contributeurs attache à l'étude des interfaces cultures régionales-développement économique. Quelques enseignements généraux tirés de cet ouvrage peuvent être formulés comme suit:

- Il n'y a pas unanimité sur la nécessité des relations économie-culture et leur intensification. Certains auteurs voient dans la culture régionale un ensemble de ressources qui peuvent être mises et doivent être mises utilement au service de l'économie; d'autres défendent, à partir d'arguments tout aussi solides mais plutôt philosophiques, l'idée que pour jouer pleinement son rôle la culture doit rester à l'écart de l'économie. C'est assez troublant de constater que les deux argumentations bien qu'opposées sont également convaincantes.

- Il n'y a pas une culture régionale mais il existe plusieurs strates de cultures régionales qui interfèrent avec l'économie régionale. C'est là le travail de Jacques Garnier sur le système productif provençal (culture provençale, culture ouvrière, culture résidentielle, culture scientifique et technique). Cette analyse montre que les formes culturelles valorisables par l'économie sont multiples, par exemple les cultures régionales immatérielles dont l'UNESCO assure la promotion.

- La culture régionale n'a cependant pas que des impacts positifs sur l'économie et le développement. Xavier Greffe l'avait déjà démontré dans plusieurs rapports sur la valorisation économique de la culture. Le travail de Michelle Bergadaà sur le Béarn et le Pays basque et un autre d'Alain Alcouffe sur le Label linguistique Oc per l'Occitan et les missions du CEEPOC ${ }^{(14)}$ confortent cette analyse.

- Le recours à la culture régionale, associée aux produits régionaux ou à l'artisanat d'art, se révèle généralement un bon investissement apprécié des firmes et des consommateurs.

\section{La place de la culture dans une société de marché}

Le problème majeur selon moi, c'est qu'une forme spécifique de culture, une métaculture, l'emporte sur toutes les autres: je veux parler du raisonnement économique fondé sur la quantification et sur un calcul, le calcul coût-avantage souvent individuel, parfois collectif visant à déterminer les actions avantageuses, voire un optimum rationnel issu de la maximisation de l'utilité ou du profit. J'ai l'impression que le raisonnement économique, ainsi défini, étend son influence et que plus grand-chose n'échappe à la rationalité économique, et donc évidemment les territoires en sont également très affectés.

Dans les approches usuelles en analyse économique, la culture n’a pas droit de cité. Les économistes se méfient considérablement de ce qu'ils nomment l'approche culturaliste. Pour un économiste, ce qui fait la force d'un modèle de développement ce sont des variables économiques, des choix stratégiques, pas la dimension culturelle. Sur les questions économiques, le raisonnement économique standard se pense auto-suffisant (les variables économiques suffisent à formuler et résoudre tout problème). 
Pourtant ces dernières années, la culture a rejoint la boîte à outils de l'économiste. Avec d'autres ressources territoriales, elle a fait son entrée dans l'analyse économique, mais c'est sous la forme de capital social et culturel (cf. les travaux de la commission Stiglitz, Sen, Fitoussi, Conseil de l'Europe, $\left.\mathrm{OCDE}^{(15)}\right)$. À partir du moment où la culture est assimilée à du capital, elle s'intègre au raisonnement économique standard et il est possible d'ouvrir le champ de l'économie de la culture et des industries culturelles. Cette intégration de la culture à l'analyse économique se déroule suivant des modalités qui placent la culture au service de l'économie.

Dans cet ouvrage, j'ai considéré que la bonne méthode ne consistait pas à envisager la culture comme une ressource économique parmi d'autres (un facteur de production, un capital) au service du développement mais davantage comme un pôle essentiel à toute civilisation et qui fait face au raisonnement économique. Autrement dit, je propose une grille d'analyse qui place d'un côté le raisonnement économique et de l'autre les autres raisonnements que j'ai qualifié d'institutionnels ou de culturels qu'ils soient guidés par des considérations politiques, philosophiques, sociales, morales, religieuses, etc.

L'une des raisons de cette séparation, c'est la suprématie actuelle du raisonnement et des préoccupations économiques. La suprématie du raisonnement économique pose des problèmes pour la pérennité des cultures régionales, car parfois le raisonnement économique est destructeur des cultures régionales (c'est du moins ce que pensent beaucoup d'auteurs). Un des résultats de ce travail c'est que l'économie et la culture doivent d'abord être dissociées pour entrer utilement en contact. De manière à déterminer quelles sont les interfaces profitables.

Avant de le montrer sur des exemples précis, je veux revenir à l'importance du raisonnement économique. Celui-ci a été fondé à l'origine par les physiocrates français puis les classiques anglais (en particulier, Adam Smith, David Ricardo et John Stuart Mill) et ensuite formalisé par le courant néo-classique qui s'est considérablement renforcé depuis la fin du XIX ${ }^{\mathrm{e}}$ siècle pour devenir au XX ${ }^{\mathrm{e}}$ et début $\mathrm{XXI}^{\mathrm{e}}$ le courant de pensée qualifié de main stream (ou pensée unique). Pas seulement dans la sphère académique mais dans la société tout entière. Tout problème actuel est d'abord envisagé sous l'angle d'une comparaison coût-avantage et d'un calcul d'optimisation. Un point doit être rappelé: c'est le fait que les fondateurs de ce mode de raisonnement (les économistes utilitaristes) avaient une tout autre idée en tête des rapports sociaux et des rapports économie-culture, qu'ils avaient une conception non égoïste et non opportuniste de l'utilitarisme. La doctrine utilitariste est donc devenue au contact de l'économie politique scientifique, méconnaissable ${ }^{(16)}$.

15 Elle distingue six types de «biens " ou capitaux: biens économiques (infrastructures, équipements, entreprises, marchés...); biens environnementaux (sous-sols, sols, ressources hydriques, biosphère - êtres vivants, biodiversité, écosystèmes -, atmosphère) ; capital humain (population, savoirs, savoirfaire...); capital social (relations humaines et liens, confiance); capital culturel (valeurs communes, connaissances - de l'histoire, des sciences, etc.); capital institutionnel et politique (institutions démocratiques, droits de l'homme, règles, formes de régulation, etc.). 
J. Stuart Mill qui a vécu et qui est mort en Avignon, qui était déjà économiste mais encore très philosophe, a écrit: "Quand la vie ne donne pas satisfaction, c'est immédiatement après l'égoïsme, à l'absence de culture qu'il faut l'attribuer, [...] ». Il rappelle aussi que l'idéal utilitariste, c'est le bonheur général et non le bonheur individuel égoïste. Or c'est une conception de l'homme et de la société très différente, suivant laquelle chacun effectue en permanence, de son seul point de vue, des arbitrages coût/avantage (et la société des calculs d'optimisation), qui s'est finalement imposée et diffusée à l'échelle de la planète. Cette rationalité individualiste, égoïste et hyper-rationaliste a tendance à envahir le champ du politique, du social, et si l'on en croit certains économistes, également de la vie familiale et de la vie en collectivité( ${ }^{17)}$. La subordination de la culture à l'économie n'est pas une idée nouvelle, c'est autant la thèse de Marx que celle de Max Weber (L'éthique protestante et l'esprit du capitalisme) et c'est aussi celle de la «regional cultural theory / l'économie régionale culturelle » dont je parle dans le livre. La culture régionale, selon cette approche, n'est utile que si elle favorise la prise de risque économique, la tolérance aux inégalités, etc. L'économie nous dit ce que doit être la culture, ce qu'elle doit apporter, au risque de s'appauvrir.

\section{Les sources d'appauvrissement et d'enrichissement de la culture}

Nous avons chacun un point de vue pour expliquer la place trop faible de la culture. Pour Philippe $\mathrm{Val}^{(18)}$, les sources de l'appauvrissement de la culture résident dans le sociologisme, dans une forme abâtardie de la culture qu'il nomme l'animation culturelle et qui consisterait à promouvoir l'expression des catégories sociales dominées, quelle que soit la qualité culturelle des œuvres. Pour d'autres, c'est le manque de moyens injectés dans le secteur culturel. Je pense pour ma part que c'est un type de raisonnement économique, certes apparemment rationnel (notamment dans une société de marché) mais trop étroit, qui appauvrit la culture et la civilisation.

J'adopte une définition très large de la culture qui inclut outre les industries culturelles, les arts, le spectacle vivant, les sciences, les cultures populaires (je me rapproche de la définition anthropologique d'André Gorz ou de Denis de Rougemont, selon laquelle la culture équivaut à l'humanité de l'Homme, à l'expérience de l'altérité). Finalement pour les besoins de ma recherche, je définis comme culturel tout comportement humain non-économique au sens où il ne résulte pas d'un calcul coût/avantage (généralement individuel) et d'optimisation sous contrainte, autrement dit, qui ne relève pas du raisonnement micro-économique standard. Pour quelle raison? Pour séparer en premier lieu le comportement individuel du comportement collectif mais surtout pour rappeler qu'il existe encore (mais de moins en moins) des institutions collectives qui fonctionnent sans faire appel au calcul et à la rationalité économique (rouler à droite, parler alsacien ou provençal, défiler le 14 juillet, etc.).

Selon moi, ce que l'on appelle culture c'est le fait d'exprimer une préférence, c'est le fait d'attacher de l'importance (un prix hors marché et non quantifiable) à certaines pratiques, à des choses matérielles ou immatérielles (valeurs, traditions). C’est le fait

17 Laurent Davezies, Le nouvel égoïsme territorial. Le grand malaise des nations, Paris, Seuil, 2015.

18 Malaise dans l'inculture, Paris, Grasset, 2015. Reprenant à contre-pied le titre du fameux livre de Freud, Malaise dans la culture - qu'il faut également relire, c'est une des dernières recommandations de Bernard Marris. 
d'accorder une valeur à quelque chose sans effectuer un calcul coût/avantage. La culture sort de la rationalité telle qu'elle est définie par l'analyse économique.

Nous connaissons bien les externalités, ou effets hors-marché qui sont la conséquence involontaire et indirecte de l'action d'un agent sur le bien-être économique des autres. En ce sens les externalités sont internalisables puisque l'on peut leur affecter un prix fictif et les réintégrer dans un calcul économique. Les choix culturels au contraire n'ont pas de prix ni d'explication économique. Ils sont plus extérieurs encore au marché que les externalités. Ce sont des super-externalités.

L'économie désignerait donc le monde du quantifiable et du calculable tandis que la culture nous renvoie à un univers non-calculable. Évidemment ces deux mondes cohabitent et interfèrent: nous connaissons les industries culturelles, les aspects culturels qui favorisent l'enrichissement, le développement, l'emploi, etc.

Il s'agit donc de mieux séparer l'économie du culturel pour ensuite voir comment ils se rapprochent, comment ils interagissent pour comprendre quels sont les rapprochements fructueux pour le dialogue et l'épanouissement des deux pôles et éventuellement quels sont les interfaces problématiques, celles qui dysfonctionnent.

\section{Quelques enseignements des interfaces souhaitables pour les territoires}

Tout le livre traite à partir d'exemples précis ou d'analyses théoriques de ces interfaces. D’une façon générale, il n’est pas possible de définir a priori les interfaces qui seraient entièrement dommageables pour le développement régional. Les stratégies très répandues actuellement de développement territorial par l'action culturelle, de valorisation des produits régionaux, etc., montrent des réussites très appréciables mais aussi des dérives et des effets pervers (gentrification, déculturation, dilapidation du patrimoine).

Dans ces interfaces problématiques, le plus souvent le raisonnement économique aplatit, exploite, réduit ou nie la dimension culturelle. Il y a problème lorsque la dimension culturelle est absente ou minorée dans les projets de développement.

Les exemples d'interfaces problématiques sont nombreux, notamment dans le marketing territorial, le «branding places " ou le «city branding», lorsque les messages sont conçus de façon trop limitée (les régions considérées du point de vue des seuls milieux d'affaires) et destinés à des utilisateurs potentiels et non pas réels, à d'hypothétiques investisseurs. Également dans la mise en œuvre de la compétitivité territoriale (cf. indice synthétique de compétitivité de la Commission européenne), lorsqu'on réduit les services publics locaux ou les salaires; dans la recomposition territoriale (redécoupage des territoires du seul point de vue économique, en oubliant la dimension historique).

Dans la publication de la synthèse de l'exercice prospective que l'Alsace vient de terminer, alors que la région Alsace est devenue une marque et qu'elle est en passe également pour des raisons économiques de se fondre dans une grande région ALCA (ACAL ou CHAMALO), il est écrit: "Réfléchir à l'horizon 2030 implique d'entrée de jeu de bien prendre la mesure du fait que l'Alsace est de plain-pied dans la globalisation, c'est-à-dire dans un environnement très concurrentiel et qui le restera. Cette réalité impose un autre cadre de réflexion $[\ldots] »{ }^{(19)}$. De façon analogue l'agence France 
Stratégie qui a étudié la réforme territoriale du point de vue de sa cohérence économique (taille des régions, déplacement domicile-travail, intensité des liens d'actionnariat) peut écrire: "Aucun département de la région ALCA ne subit de force centrifuge, elle est donc parfaitement cohérente. Les déplacements de travailleurs comme les liens financiers sont relativement limités au sein de la région comme vis-à-vis des autres régions ${ }^{(20)}$.

On voit là que l'analyse économique, aujourd'hui dominée par l'école autrichienne et la pensée néolibérale (la mondialisation, la concurrence, l'exigence d'attractivité, de compétitivité, de réduction de la dépense publique, etc.), impose aux régions des adaptations (se redimensionner, être compétitive suivant les critères de l'UE ou des milieux d'affaires), des mutations, dans le cadre d'un raisonnement autonome, en s'affranchissant de toute autre considération. Cela ne concerne pas que les sept nouvelles grandes régions, issues du regroupement d'anciennes régions, mais aussi les six régions dont le périmètre reste inchangé dont Provence-Alpes-Côte d'Azur.

Il y a des interfaces heureuses. Mes collègues en ont proposé plusieurs: le rapport entre le patrimoine linguistique et le développement local, entre la créativité, l'innovation et le territoire, entre les produits régionaux artisanaux et le territoire ${ }^{(21)}$. Là encore il n'y a pas de recette miracle. Chaque projet de valorisation de l'héritage culturel régional (à travers des opérations ponctuelles comme L'Hermione ou durables comme le MUCEM) va susciter des partisans et des détracteurs. Il y a cependant quelques règles simples comme la concertation de la population, une bonne relation créateurgestionnaire dans l'organisation des projets qui laisse chacun libre dans son domaine (cela suppose le respect mutuel et le dialogue), un ancrage territorial fort, la tolérance vis-à-vis de l'innovation et la créativité en matière de développement (les modèles régionaux de développement comme la culture régionale doivent pouvoir évoluer), une connaissance suffisante de la région mais aussi de la science régionale et des modèles régionaux de développement. Cette interface heureuse, je l'appelle: «culture du développement ». Ce sera ma conclusion provisoire.

\section{Pour conclure provisoirement: le concept de culture du développement}

Le développement territorial n'est pas simplement le fait de bénéficier d'une conjoncture économique favorable dans une région donnée à un moment donné. On a vu au cours de l'histoire des régions en grande difficulté qui ont su tirer parti d'une situation initialement très défavorable (ex: les Pays-Bas au XVII ${ }^{e}$ siècle qui ont su inventer des techniques pour surmonter leurs handicaps territoriaux: la construction de digues, de polders, l'augmentation de la productivité agricole, l'organisation essentiellement urbaine, - plus proche de nous dans les années 90, la création du Musée Guggenheim à Bilbao en pleine débâcle économique).

Je pense de plus en plus que le développement territorial est un art collectif et un métier qui exige des compétences économiques et culturelles et de dialogue économieculture. La mise en œuvre du développement à l'échelle régionale requiert un modèle

20 Arno Amabile, Claire Bernard et Anne Épaulard, France Stratégie, Réforme territoriale et cohérence économique régionale, 2015, p. 6.

$21 \mathrm{KAHN} / \mathrm{Le}$ SQUère/Kosianski, Cultures régionales (note 13). 
de développement pensé sur place et non un modèle importé, une concertation aussi large que possible ainsi qu'une connaissance fine du territoire, de sa géographie, de son histoire, de sa population, de ses entreprises, etc. Cela s'apprend sur le terrain comme à travers des formations et dans la littérature régionale. Cela prend du temps.

\title{
Résumé
}

La pensée et la vie économique contemporaines sont très largement dominées par le dogme de l'école néoclassique et néolibérale autrichienne suivant lequel l'organisation sociale peut être entièrement comprise à partir de deux hypothèses: la société se réduit à l'économique et les activités économiques sont exclusivement expliquées par la rationalité utilitariste des individus. Cette approche rend inutile, au moins dans la sphère économique, la constitution d'un cadre culturel collectiffondé sur des préférences et des valeurs spécifiques à une communauté située. Or un grand nombre de travaux pluridisciplinaires confirment le bien-fondé des observations de Karl Polanyi et le maintien de la dimension culturelle, au cour de l'activité économique, notamment dans l'entreprise et dans les territoires. Le présent travail commente les apports de deux ouvrages collectifs : le premier consacré à l'entreprise rhénane et le second dédié aux relations entre les cultures régionales et le développement économique. En s'appuyant sur la littérature qui lui est consacré, l'article interroge la nature du modèle rhénan. Il montre également comment les cultures régionales parviennent à enrichir la sphère économique, en dépassant l'individualisme méthodologique, pour construire une culture territoriale $d u$ développement.

\begin{abstract}
Contemporary economic thought is overwhelmingly dominated by the dogma of the neoclassical school and the Austrian neoliberal school, according to which social organization can fully be understood from two hypotheses: the society can be reduced to its economic aspects, and economic activities are exclusively explained by the utilitarist rationality of individuals. This approach makes unnecessary, at least in the economic sphere, the construction of a collective cultural framework based on preferences and values specific of a "situated" community. Now, many multidisciplinary works confirm the validity of Karl Polanyi's observations and the embedment of cultural aspects in the economic activity, especially within the firms and the territories. This paper discusses the contributions of two collective works: the first book devoted to the Rhine area's model of firm; the second devoted to the relationships between regional cultures and economic development. Based on the literature on this subject, the paper questions the nature of such a model. It also shows how regional cultures succeed to enrich the economic sphere, beyond the methodological individualism, through the construction of a culture of development.
\end{abstract}

